\title{
Night as frontier
}

Night as Frontier

\section{Murray Melbin}

\section{(2) OpenEdition}

1 Journals

\section{Édition électronique}

URL : http://journals.openedition.org/conflits/19434

DOI : 10.4000/conflits. 19434

ISSN : $1777-5345$

Éditeur :

CCLS - Centre d'études sur les conflits lilberté et sécurité, L'Harmattan

\section{Édition imprimée}

Date de publication : 15 juillet 2017

Pagination : 29-59

ISBN : 978-2-343-12713-2

ISSN : 1157-996X

Référence électronique

Murray Melbin, « Night as frontier », Cultures \& Conflits [En ligne], 105-106 | printemps/été 2017, mis en ligne le 15 juillet 2019, consulté le 30 mars 2021. URL : http://journals.openedition.org/conflits/19434 ; DOl : https://doi.org/10.4000/conflits.19434 


\section{Night as frontier 1}

\section{Murray MELBIN 2}

Murray Melbin est sociologue, professeur émérite de l'Université de Boston. Il est notamment l'auteur de Alone and with others. A grammar of interpersonal behavior (1972). Nous traduisons ici l'un de ses textes, paru en 1978 dans l'American Sociological Review, qui préfigure son livre Night as frontier. Colonizing the world after dark, publié en 1986. Il y fait le constat d'une "conquête » de la nuit, qui correspond à une extension temporelle des activités humaines dans les sociétés industrialisées, et s'interroge, avec les méthodes de la sociologie expérimentale, sur la spécificité des comportements sociaux la nuit. Ce texte présente un intérêt documentaire (sur le développement des activités nocturnes an XXe siècle aux ÉtatsUnis) mais ouvre aussi une réflexion sur la dialectique d'une nuit qui est à la fois un espace-temps singulier et un prolongement des structures économiques et sociales du jour.

T es hommes semblent voués à accomplir de plus en plus d'activités à toute Lheure du jour et de la nuit. Ces activités sont très variées, concernent un très large public, et la tendance est mondiale. Une hypothèse pour en rendre compte de manière globale consiste à appréhender la nuit comme une nouvelle frontière, au sens où l'expansion dans les heures sombres serait la continuation des migrations géographiques. Pour défendre ce point de vue, je documenterai cette tendance puis présenterai une hypothèse sur la nature du temps

1. NDT : «Night as Frontier " a été publié en 1978 dans l'American Sociological Review (vol. $43, n^{\circ} 1$, pp. 3-22). Nous avons préféré conserver le titre dans sa version originale tant le concept de frontière est spécifique aux États-Unis. Nous avons, selon les cas, traduit «frontier» par frontière, conquête, front pionnier, colonisation ou confins, pour privilégier la qualité du texte en français. La traduction du texte a été réalisée par Thomas Alam, Anne-Cécile Douillet et Nicolas Kaciaf.

2. Je remercie le Center for Studies of Metropolitan Problems et le National Institute of Mental Health pour le financement $\mathrm{MH}-22763$, qui a rendu possible la recherche et la rédaction de cet article, et Earl Mellor, du Burean of Labor Statistics, pour m'avoir fourni les bases de données du Current Population Survey de 1976 et m'avoir aidé à les interpréter. Je remercie aussi mes assistants de recherche - William O. Clarke, Ann Getman, Shelley Leavitt, Lee Parmenter, Alan Rubenstein, Melanie Wallace et Marilyn Arsem - pour les observations de terrain qu'ils ont réalisées, à toute heure, sous la pluie et le froid intense ou sous une météo plus clémente, et mes collègues Paul Hollander et Anthony Harris, de l'Université du Massachusetts à Amherst, pour avoir accepté de jouer le rôle de destinataire dans le test de la clé perdue. 
et sa relation à l'espace. En troisième lieu, je montrerai que la vie sociale nocturne présente beaucoup de caractéristiques analogues à celles de la vie sur les fronts pionniers.

\section{Le processus d'expansion}

Nous étions autrefois une espèce diurne, menant nos activités entre l'aube et le crépuscule. Apprenant à maîtriser le feu, les premiers hommes l'ont utilisé pour cuisiner mais aussi pour des moments de convivialité pouvant durer plusieurs heures après la tombée de la nuit. Il leur arrivait aussi de s'affairer jour et nuit. On observe ainsi au cours des siècles des feux de camp militaires, des veillées de prière dans les temples, des cérémonies de fiançailles à minuit, des sentinelles qui gardent les portes des villes, des matelots qui font le guet sur les navires, des cérémonies de guérison chez les Indiens vénézuéliens qui s'étalent de la tombée de la nuit au lever du soleil, des aubergistes qui servent les voyageurs à toute heure. Au cours du premier siècle après Jésus-Christ, Rome a été obligée de restreindre les déplacements de chariots pendant la nuit pour limiter les embouteillages ${ }^{3}$.

Pour autant, les activités en continu sont restées marginales jusqu'au XIXe siècle. C'est à cette époque que l'étendue et le rythme des périodes d'activités ont considérablement augmenté. William Murdock a développé une méthode pratique d'éclairage au gaz de houille et, en 1803, a tout mis en œuvre pour que l'intérieur de l'usine de Soho, à Birmingham, en Angleterre, soit ainsi éclairé. D'autres manufactures des alentours ont alors commencé à utiliser l'éclairage au gaz. Des méthodes pour distribuer le gaz de houille à tous les bâtiments et lampadaires des villes ont été introduites peu de temps après. En 1820, Pall Mall à Londres a été la première rue à être éclairée par le gaz de houille. L'éclairage artificiel a très fortement stimulé l'industrie du divertissement ${ }^{4}$. Cela a aussi rendu possible une rotation beaucoup plus importante des équipes dans les usines. Ainsi, dès 1867, Karl Marx ${ }^{5}$ déclarait que le travail de nuit était une nouvelle forme d'exploitation de la main d'œuvre.

Aux États-Unis, dans les dernières décennies du XIXe siècle, le déplacement des migrations humaines de l'espace vers le temps a résulté de deux dynamiques. En 1890, le Bureau du recensement annonçait que la conquête de l'Amérique était terminée, dans la mesure où il n'était plus possible de dessiner une ligne continue sur la carte de l'Ouest américain pour marquer les avancées de la colonisation. Parallèlement, la recherche du matériau optimal pour éclairer les lanternes et produire de la lumière blanche de manière continue culminait en 1885 avec l'invention du manchon à incandescence

3. Mumford L., The City in History, New York, Harcourt Brace, 1961, p. 217.

4. Schlesinger A., The Rise of the City: 1878-1895, New York, Macmillan, 1933, p. 105.

5. Marx K., Capital, New York, Modern Library, 1906 [1867], chap. 10, sec. 4. 
Welsbach, une maille de coton imprégnée de produits chimiques. L'utilisation des heures sombres continua à augmenter, et s'accrut encore avec l'apparition de l'éclairage électrique.

Différentes publications documentent cette tendance. Pendant la Première Guerre mondiale émerge ainsi une préoccupation spécifique, exprimée par Louis Brandeis et Josephine Goldmark ${ }^{6}$ dans The Case Against Night Work for Women, à propos de l'impact du travail à horaires spéciaux. Une dizaine d'années plus tard, en 1927, le National Industrial Conference Board ${ }^{7}$ publie une enquête très complète sur les caractéristiques des travailleurs de nuit.

L'indice le plus systématique de l'augmentation continue des activités 24h/24 aux États-Unis est la croissance des programmes radiophoniques et télévisuels. En effet, les diffuseurs ont développé des enquêtes marketing pour planifier leurs programmes et établir les tarifs publicitaires. Le nombre des stations actives à certaines heures et l'extension de ces heures tout au long de la nuit reflètent les estimations de l'époque concernant l'ampleur de la population éveillée et le nombre d'auditeurs potentiels. Le tableau 1 montre une tendance à l'extension des horaires de diffusion, jusqu'à la programmation en continu, à la radio comme à la télévision. Bien que cela n'apparaisse pas dans le tableau, les programmes de la télévision à Boston se terminaient à $23 \mathrm{~h} 30$ en 1949, puis ses horaires se sont étendus pour inclure le Late Show et ensuite le Late Late Show dans les années suivantes, jusqu'en 1974. Chaque média a adopté progressivement une programmation $24 \mathrm{~h} / 24$, reflétant la croissance des activités nocturnes.

C'est seulement au cours de la dernière décennie [les années 1970] que le U.S. Burean of Labor Statistics ${ }^{8}$ a commencé à poser des questions sur les moments de la journée pendant lesquels les gens travaillent. En 1976, sur une population active de 75 millions de personnes, 12 millions ont indiqué qu'elles travaillaient essentiellement pendant la nuit, dont 2,5 millions soumises à des horaires de travail débutant à minuit. Dans la mesure où ces données n'incluent pas la clientèle qui fréquente les établissements nocturnes tels que les restaurants, les services d'urgence hospitaliers, les casinos et cercles de jeu ou les transports publics, elles constituent une approximation basse du nombre de personnes debout la nuit. Aujourd'hui plus qu'avant, des tas de gens s'activent hors de leur domicile à toute heure du jour et de la nuit et dans des domaines très variés. Il y a les supermarchés $24 \mathrm{~h} / 24$, les bowlings, les grands

6. Brandeis L. D. et Goldmark J., The Case Against Night Work for Women, New York, National Consumers League, 1918.

7. National Industrial Conference Board, Night Work in Industry, New York, National Industrial Conference Board, 1927. NDT : Il s'agit d'un think tank fondé en 1916 et financé par le patronat pour produire une expertise en matière de relations salariales.

8. U.S. Bureau of Labor Statistics, Current Population Survey. Unpublished paper, Washington, D.C, May 12 1976, Table 1. 
magasins, les restaurants, les cinémas, les ateliers de réparation automobile, les services de taxis, les terminaux de bus et d'avions, les chaînes de radio et de télévisions, les agences de location de voiture et les stations-service. Il y a aussi les usines de raffinage qui fonctionnent $24 \mathrm{~h} / 24$, les usines opérant en $3 \times 8$, le service postal, les journaux, les hôtels et les hôpitaux. Certains services fonctionnent en continu : la fourniture d'énergie et d'électricité, les péages routiers, les patrouilles de police et les services téléphoniques. Il y a aussi tout un tas de services d'urgence et de réparation qui fonctionnent avec des systèmes de garde : les pompiers, les dépanneurs, les serruriers, les fournisseurs de langes propres, les ambulanciers, les prêteurs sur gage, les désinsectiseurs, les réparateurs de télévision, les vitriers et les pompes funèbres. La tendance à l'expansion de la vie nocturne est engagée en dehors des États-Unis aussi. En Grande-Bretagne, depuis la Seconde Guerre mondiale, la croissance annuelle de la force de travail manuelle sur les horaires de nuit à l'usine a été de 1 \% par an, et les taux d'augmentation ont été bien plus importants chez les constructeurs automobiles et dans l'industrie chimique ${ }^{9}$. Richard Meier ${ }^{10}$ observe que Singapour est en train de devenir la ville qui vit le plus intensément 24h/24. Des données sur l'activité nocturne au Pérou, en France, en URSS et dans huit autres pays sont par ailleurs disponibles dans un ouvrage sur l'usage du temps ${ }^{11}$.

\begin{tabular}{|c|c|c|c|c|c|c|c|c|c|c|}
\hline & \multicolumn{10}{|c|}{ Étendue de la programmation commerciale } \\
\hline & Avril & Avril & Avril & Avril & Avril & Avril & Avril & Avril & Avril & Avril \\
\hline & 1929 & 1934 & 1939 & 1944 & 1949 & 1954 & 1959 & 1964 & 1969 & 1974 \\
\hline & \multicolumn{10}{|c|}{ Radio } \\
\hline $\begin{array}{c}\text { Nombre } \\
\text { de stations }\end{array}$ & 7 & 7 & 8 & 7 & 8 & 14 & 15 & 20 & 26 & 27 \\
\hline $\begin{array}{c}\text { Nombre de } \\
\text { stations } 24 / 24\end{array}$ & 0 & 0 & 0 & 0 & 0 & 1 & 3 & 8 & 12 & 15 \\
\hline \multirow[t]{2}{*}{$\begin{array}{l}\text { Proportion de } \\
\text { stations } 24 / 24\end{array}$} & / & / & / & / & / & $7 \%$ & $20 \%$ & $40 \%$ & $46 \%$ & $57 \%$ \\
\hline & \multicolumn{10}{|c|}{ Télévision } \\
\hline $\begin{array}{c}\text { Nombre } \\
\text { de chaînes }\end{array}$ & / & / & ' & / & 2 & 4 & 4 & 4 & 5 & 7 \\
\hline $\begin{array}{l}\text { Nombre de } \\
\text { chaînes } 24 / 24\end{array}$ & / & / & / & / & 0 & 0 & 0 & 0 & 0 & 1 \\
\hline $\begin{array}{l}\text { Proportion de } \\
\text { stations } 24 / 24\end{array}$ & I & I & 1 & I & I & 1 & I & I & I & $14 \%$ \\
\hline
\end{tabular}

Tableau 1. Nombre de stations de radio et de chaînes de télévision et heures de programmation à Boston

Sources : Données issues des journaux de Boston - Globe, Herald, Record et Traveler - et des diffuseurs eux-mêmes. Si l'on observe des divergences dans les horaires de diffusion en AM et en FM pour une même station de radio ou dans les horaires de diffusion en VHF et en UHF pour une même chaîne de télévision, alors la programmation est considérée comme relevant de deux stations ou chaînes. 


\section{La colonisation de l'espace et du temps}

Le temps, comme l'espace, permet de caractériser la niche écologique des espèces. Bien que les appropriations du cycle nycthéméral soient très variées d'une espèce à l'autre, nous nous contentons de les qualifier par les termes « diurne » et «nocturne », qui renvoient à la période pendant laquelle les créatures sont actives. Or, nous améliorons notre connaissance de l'écosystème d'une région en observant l'activité nocturne des ratons laveurs, des chouettes et des rats, tout en étudiant la dispersion spatiale de ces animaux et bien d'autres. Par ailleurs, une portion de forêt, de prairie ou de récif corallien est utilisée de manière continue par des créatures diurnes et nocturnes qui se relaient activement pour l'occuper. Nous opérons le même type de classements géographiques à propos des groupes humains. Nous distinguons ainsi des peuples insulaires, des peuples du désert ou encore des peuples des terres arctiques, pour caractériser leur environnement.

Le traitement identique du temps et de l'espace repose sur le postulat selon lequel tous deux sont constitutifs du cadre de vie. Un bon exemple en est la définition du mot occuper [occupy] dans le dictionnaire : " 2. Remplir (prendre du temps ou de l'espace) : une conférence qui a occupé trois beures 12 ». Les géographes étudient davantage les activités que les structures physiques pour déterminer si et comment les gens occupent l'espace ${ }^{13}$. La seule présence de bâtiments, et autres structures matérielles, dans des endroits comme le Machu Picchu, Petra et le Zimbabwe ne suffit pas à nous convaincre que ces lieux sont habités. Les anciens centres miniers de l'Ouest Américain, autrefois très animés, ne sont plus des colonies mais des villes fantômes. Inversement, on peut dire qu'une région agricole dans laquelle des gens sont actifs est habitée, bien que les bâtiments soient rares. En d'autres termes, la présence de structures construites par l'homme n'est pas un critère d'occupation d'une région, à l'inverse des gens et de leurs activités.

De la même façon que l'occupation de l'espace est éparse dans les colonies rurales, l'occupation du temps peut ne pas être très dense. Par exemple, London Transport propose vingt-et-une lignes de bus toute la nuit. Sur beaucoup de ces lignes, le service nocturne correspond à un bus par heure. Pour autant, même si le bus ne passe pas pendant les cinquante-neuf autres minutes, le dispositif est présenté comme continu. Si un moment actif est entouré de moments calmes, l'ensemble de la période est pensé comme occupé.

9. Young M. et Willmott P., The Symmetrical Family, Harmondsworth, England, Penguin, 1973, p. 175.

10. Meier R. L., "A stable urban ecosystem », Science, vol. 192, n4243, 1976, p. 965.

11. Szalai A. (ed.), The Use of Time, La Hague, Mouton, 1972, annexes.

12. American Heritage Dictionary of the English Language, Boston, Houghton Mifflin, 1970, p. 908.

13. Buttimer A., "Grasping the dynamism of lifeworld ", Annals of the Association of American Geographers, n66, 1976, p. 286. 
Évidemment, le temps est toujours utilisé en relation avec un espace donné. Réciproquement, l'espace est toujours pratiqué en conjonction avec certaines heures de la journée. Ainsi, l'espace et le temps forment ensemble le contenant de l'activité humaine. Nous avons oublié cet aspect dans le cas des anciennes conquêtes de l'Ouest parce que l'expansion spatiale a été spectaculaire. Beaucoup moins d'attention a été portée aux seize heures d'éveil car la durée quotidienne d'activité était relativement constante à mesure que l'expansion géographique se développait. Comme l'usage du temps est resté inchangé, cet élément a été ignoré dans les théories de l'écologie humaine. De nos jours, l'expansion se déroule dans le temps. Dans la mesure où les gens sont susceptibles d'exploiter une niche à travers une distribution de leurs activités sur un éventail plus large d'heures tout autant qu'à travers l'occupation de l'espace, un front pionnier peut aussi apparaître dans la dimension temporelle.

Une colonie peut être définie comme une occupation stable de l'espace et du temps par les hommes et leurs activités. Un front pionnier est un ensemble de colonies clairsemées dans l'espace et le temps, situé entre une région densément peuplée et une région pratiquement vide. En dessous d'une certaine densité d'individus actifs, une région donnée de l'espace-temps est considérée comme un désert. À mesure que la densité s'élève à partir de ce point, la présence d'individus actifs autorise à qualifier cette zone de frontière. Au-delà de cette deuxième ligne de démarcation, une plus grande densité de personnes actives transforme cette zone en une région habitée. Dans une période historique donnée, les limites du front pionnier peuvent être stables ou en expansion. Son expansion implique de s'aventurer dans l'inconnu et s'accompagne souvent de nouveautés et de changements.

\section{Les similarités entre frontières terrestres et frontières temporelles}

Deux arguments sous-tendent l'hypothèse de la nuit comme frontière. Le premier est que les forces qui président à l'expansion de l'activité dans les heures sombres sont les mêmes que celles qui président à l'expansion territoriale. Autrement dit, la même explication causale devrait rendre compte de l'étalement des gens et de leurs activités, que ce soit dans l'espace ou dans le temps. J'en ai esquissé une analyse dans un autre article; les facteurs à l'œuvre incluent le jeu de l'offre et de la demande et les effets de rétroaction ${ }^{14}$. L'autre argument serait que les mêmes traits saillants de la vie sociale se retrouvent dans les conquêtes terrestres et temporelles. La rapide expansion des activités nocturnes s'est essentiellement déroulée dans les zones urbaines. Aussi la culture de la nuit urbaine contemporaine devrait-elle révéler les mêmes caractéristiques que celle des fronts pionniers terrestres du passé. 
J'ai choisi de revisiter la vie dans l'Ouest états-unien du milieu du XIXe siècle à l'aune de la nuit d'aujourd'hui. Il y a bien sûr eu d'autres fronts pionniers terrestres et l'hypothèse devrait aussi s'appliquer à eux. Néanmoins, il y a de bonnes raisons de commencer la démonstration avec l'Ouest américain. Tout d'abord, les archives relatives à ce flux vers l'Ouest sont très fournies, bien organisées et disponibles immédiatement. Ensuite, l'Ouest américain présente une certaine continuité avec l'expansion dans la nuit. Le mouvement vers l'Ouest a atteint la côte californienne. Depuis, les principales villes de Californie sont devenues des zones d'intenses activités nocturnes, comme si le flux à travers le continent avait été dévié sur la nuit plutôt que de se déverser dans l'océan.

La frontière terrestre dont nous discutons ici est la zone à l'ouest du fleuve Mississippi pendant les décennies du milieu du XIXe siècle, entre 1830 et 1880. La nuit urbaine est ici celle de toute zone urbaine majeure, sur le créneau s'étalant de minuit à 7h30, pendant les décennies 1960 et 1970. La plupart de mes exemples sont issus d'une récente étude à Boston. En bien des aspects, la vie sociale nocturne est similaire à la vie sociale sur d'autres fronts pionniers.

\section{Une avancée par séquences}

La colonisation d'une nouvelle région procède d'une succession d'étapes. Les gens se sont aventurés aux confins de l'Ouest « par vagues successives : le chasseur et le négociant en fourrure qui se sont engagés sur le territoire indien ont été suivis par l'éleveur de bétail puis par l'agriculteur pionnier ${ }^{15}$ ». Les modes de vie étaient spécifiques à chaque étape également. Les chasseurs et les trappeurs ne se sont pas installés, à la différence des mineurs qui les ont suivis, et ces derniers vivaient encore différemment des fermiers pionniers qui sont arrivés par la suite ${ }^{16}$. Bien que les conditions de vie fussent généralement très rudes, le confort s'est sensiblement amélioré pour les fermiers qui se sont implantés durablement en comparaison des trappeurs qui se déplaçaient souvent. De même, il y a eu une succession de phases dans la colonisation de la nuit. À chaque étape, la nuit est occupée de manière plus dense et ces heures sont utilisées de manière différente. Les premiers arrivés dans les rues furent les vagabonds, puis des groupes liés à des activités de production, les équipes de nuit. Ce fut ensuite le tour de ceux qui pratiquent ou proposent des activités de consommation, les patrons de restaurants et de bars ouverts toute la nuit, et les joueurs qui se réunissent régulièrement vers minuit autour des tables de jeu. Les taux de progression sont irréguliers dans les deux cas.

14. Melbin M., "The colonization of time », in Carlstein T., Parkes D. et Thrift N. (eds.), Timing Space and Spacing Time in Social Organization, London, Arnold, 1977.

15. Turner F. J., America's Great Frontiers and Sections. Unpublished essays edited by W. R. Jacobs, Lincoln, Nebraska University Press, 1969 [1965], p. 59 et Turner F. J., The Frontier in American History, New York, Holt, 1920 [1893], p. 12 et p. 19-20.

16. Billington R. A., Westward Expansion, New York, Macmillan, 1949, pp. 4-5. 
Croissance démographique et développement ne sont pas continus. À l'Ouest, la croissance économique était erratique. Périodes de dépression, saisons sèches, et autres difficultés conduisaient beaucoup de gens à abandonner leur propriété pour retourner à l'Est. De manière similaire, avec l'embargo sur le pétrole de 1973-1974, l'activité nocturne s'est ralentie dans la mesure où certains restaurants, stations-service et autres établissements diminuaient les heures de service du public.

\section{Une population clairsemée mais aussi plus homogène}

Au début, seules quelques personnes s'aventurent dans la nouvelle région. La ligne frontière de l'Ouest américain a été dessinée par le bureau du recensement (Census bureau) à partir d'une zone de densité allant de deux à six habitants par mile carré. L'autre côté de la ligne était qualifié de « désert ». D'un point de vue démographique, le front pionnier occidental était essentiellement fait de jeunes gens vigoureux avec, proportionnellement, moins de femmes et de personnes âgées que dans les populations des États de l'Est ${ }^{17}$. Ce portrait démographique convient parfaitement à la nuit : il y a moins de gens dehors et ce sont pour l'essentiel des hommes jeunes. Une comparaison grossière entre une ligne de frontière occidentale du XIX ${ }^{\mathrm{e}}$ siècle et une période nocturne du XXe siècle peut être faite en parcourant la carte de la figure 1 de droite à gauche et le graphe de la figure 2 de gauche à droite. Les deux figures montrent des densités échelonnées de façon similaire. Dans la figure 2, la période entre minuit et 7 heures est la plus clairsemée et le rapport entre sa densité et celle du reste la journée est comparable à celui entre les régions à l'Ouest et à l'Est du Mississippi dans la figure 1. Les données montrent aussi que la proportion d'hommes dans la population est plus grande sur les fronts pionniers. Tout comme les hommes constituent la population la plus importante des États des Grandes Plaines et des Montagnes, ils représentent aussi l'essentiel de la population de la rue (89\%) au milieu de la nuit.

Une estimation de l'âge des passants a aussi été effectuée pendant les observations de terrain qui ont permis la collecte des données de la figure 218 . Alors que des gens de tous âges étaient dans la rue pendant la journée, aucun passant âgé de plus de 59 ans n'a été vu entre minuit et cinq heures du matin : et personne de plus de 41 ans entre deux et cinq heures du matin.

17. Riegel R. E, America Moves West, New York, Holt, 1947, p. 624 ; Godkin E., « The frontier and the national character ", in Ridge M. et Billington R. A. (eds.), America's Frontier Story, New York, Holt, 1969 [1896], p. 13 ; Dick E., The Sod-House Frontier, 1854-1890, New York, Appleton-Century, 1954 [1937], p. 7 et p. 232.

18. La corrélation entre l'estimation de l'âge par l'enquêteur et la réponse à la question " quel âge avez-vous ? » posée à 696 passants (sur deux ans) est de 0,96 pour les 6 observateurs (le plus petit coefficient par observateur est de 0,93). Les populations de ces sites sont relativement plus jeunes que la moyenne du recensement de la ville. 


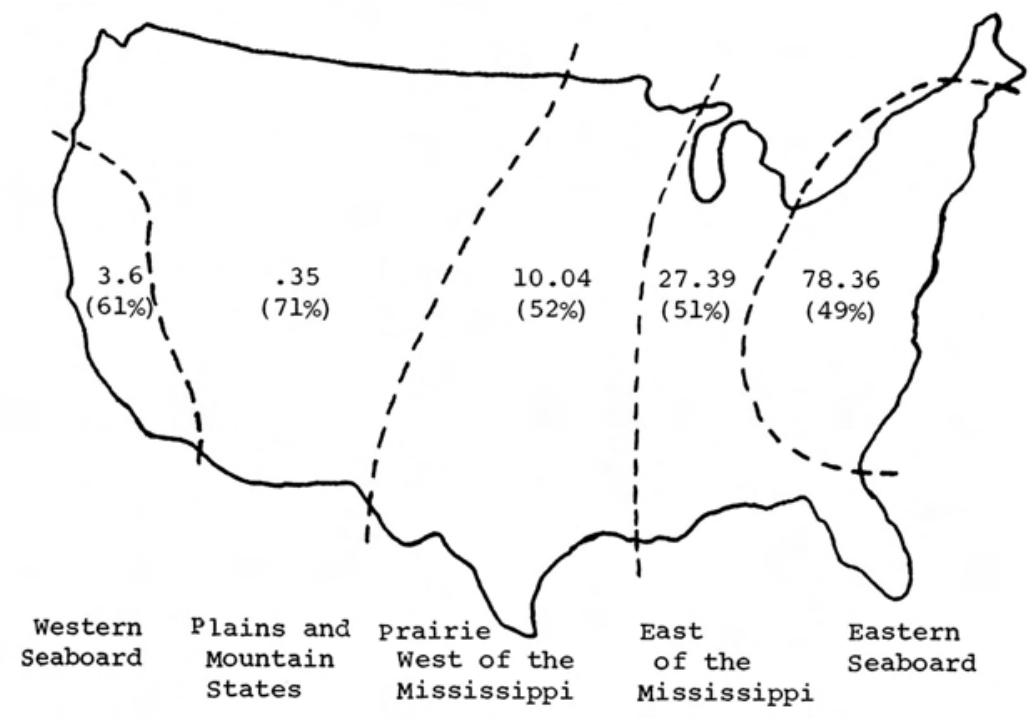

Figure 1. Habitants par mile carré (et pourcentage d'individus de sexe masculin par mile carré) au cours des décennies 1870-1880

Source : US Bureau of the Census, Historical Statistics of the United States, Washington, D.C. U.S. Government Printing Office, 1975, Vol. 1: ser. A195-209.

3. Une solitude appréciée, des contraintes sociales moins pesantes et moins de persécution

Les terres de l'Ouest, au moment de la conquête, offraient tranquillité et soulagement, loin des sentiments d'oppression. "Les négociants en fourrure [...] étaient d'un type psychologique particulier, au sens où ils trouvaient la solitude de la forêt plus acceptable que la compagnie de leurs congénères 19 ». S'échapper dans la nature sauvage avait quelque chose d'attirant, et permettait de laisser derrière soi la tromperie et les ennuis, les devoirs contrariants ou les demandes importunes du gouvernement ${ }^{20}$. «Oh, comme il est agréable le calme de ces lieux, libérés des troubles et de la perplexité de la triste Europe 21 ", écrit William Penn depuis son refuge dans la forêt. Même plus tard, l'Ouest demeurait « un refuge [...] pour les jeunes assujettis aux plus âgés 22 ». L'extrême périphérie offrait aussi la possibilité d'échapper à la persécution. C'est ainsi que les Mormons, tout comme les Huttérites, ont fait route vers l'Ouest dans l'espoir de ne plus être harcelés.

19. Billington R. A., 1949, op. cit., p. 4.

20. Robbins R. M., Our Landed Heritage, Princeton, Princeton University Press, 1942, p. 148.

21. Turner F. J, 1920 [1893], op. cit., p. 262.

22. Turner F. J., The Significance of Sections in American History, New York, Holt, 1932, p. 25. 
Total

per

$\begin{array}{lllllllllllll}4.12 & 4.52 & 6.50 & 6.71 & 6.85 & 5.11 & 3.85 & 3.46 & 1.32 & .28\end{array}$

minute

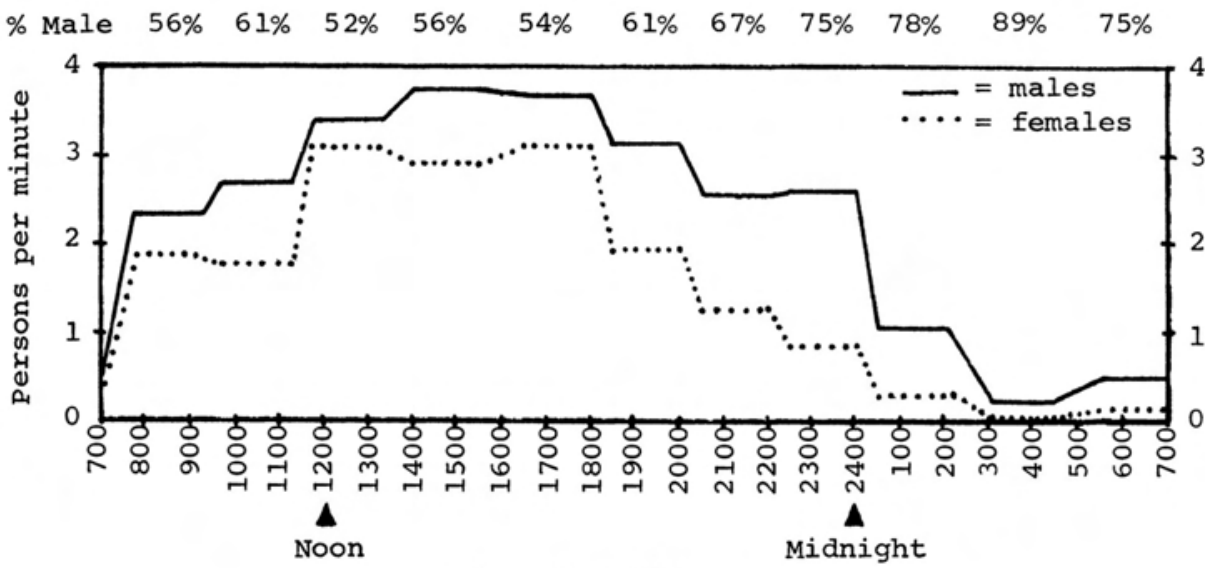

24 HOUR CLOCK

Figure 2. Femmes et hommes recensés par minute dans les rues du Centre de Boston

Les pointages ont été accomplis durant des visites de terrain dans la ville de Boston en 1974, à partir d'un échantillon stratifié aléatoire. En plus du décompte individuel, d'autres mesures ont été réalisées durant ces visites de terrain, notamment l'expérience décrite plus loin dans cette section. Les 166 visites de terrain (56 début juin, 56 début septembre et 54 début décembre), de deux heures chacune, ont été réparties sur trois sites : une rue commerçante, une rue résidentielle et une plate-forme de correspondance de transports $(62,42$ et 62 visites respectivement), chaque site étant à une distance d'un mile de l'autre sur une ligne traversant le centre-ville. Au total, nous avons réalisé 456 pointages de 5 minutes pour décompter les passants qui se déplacent dans une direction donnée, sur 8 points de passage.

Les horaires des visites de terrain ont été sélectionnés de manière aléatoire à partir d'intervalles de deux heures sur le cycle de 24 heures. J'ai établi onze intervalles dans cet échantillonnage pour deux raisons : certaines heures de la journée sont clairement associées à des activités spécifiques, notamment la tranche 7h30-9h29 (heure de pointe du matin) et celle de 16h15-18h14 (heure de pointe du soir). Étant donné le positionnement des deux créneaux de pointe sur une période de 24 heures, et étant donné la nécessité de visites de terrain de deux heures pour réaliser l'expérience et mener d'autres études associées à un programme de recherche plus général sur les activités et le temps, il a été impossible de répartir aussi précisément le reste des heures de la journée sur des intervalles de deux heures tout au long du cycle nycthéméral. Si l'on avait inséré douze intervalles de deux heures, certaines strates auraient été déphasées par rapport aux cycles temporels connus à Boston. Aussi, onze strates ont été établies et les quelques minutes restantes ont été exclues de l'échantillon de la manière suivante : heure de pointe du matin =07h30-09h29; jour =09h30-11h29, 11h30-13h29 (13h30-13h44 exclus), 13h45-15h44 (15h45-16h14 exclus); heure de pointe du soir = 16h15-18h14; soirée $=18 \mathrm{~h} 15-20 \mathrm{~h} 14,20 \mathrm{~h} 15-22 \mathrm{~h} 14,22 \mathrm{~h} 15-00 \mathrm{~h} 14$; et nuit = 00h15-02h14 (02h15-02h44 exclus), 02h45-04h44 (04h45-05h14 exclus), 05h15-07h14 (07h15-07h29 exclus). Ainsi, les deux grandes phases du jour et de la nuit perdent les minutes non échantillonnées, 45 minutes le jour et 75 minutes la nuit. Si l'on enlève ces totaux de l'échantillon, le jour, le soir et la nuit comptent chacun six heures, contre deux heures pour chaque période de pointe (Cela aurait pu être plus simple de construire un échantillon pour des périodes de douze heures puis de réaligner, au moment de l'analyse, les bornes de chaque strate temporelle sur les schémas temporels locaux. Si cette option avait été retenue, I'enregistrement des mesures aurait dû se faire pour chaque minute.) Le nombre de visites pour les cinq phases du nycthémère étaient les suivantes : heure de pointe du matin, 13 ; jour, 4 ; heure de pointe du soir, 11 ; soirée, 48 ; nuit, 47 . Les dénombrements pour chaque période de temps ont été divisés par le nombre de minutes prises en compte pour cette période afin d'égaliser les fractions échantillonnées par strates, et ensuite répliqués pour obtenir des évaluations plus sûres.

Trois équipes mixtes de chercheurs (homme-femme) - avec remaniement systématique des équipes suivant les périodes horaires et les sites - ont participé aux pointages et à l'expérience évoquée plus loin dans cette section 
De manière similaire, beaucoup d'entre nous apprécient l'expérience de marcher la nuit dans des rues qui sont ordinairement noires de monde. Les individus qui veillent tard expriment un sentiment de soulagement par rapport à l'étouffement et à l'anonymat de la vie urbaine diurne. Le calme qui caractérise ces heures est particulièrement attirant pour les jeunes, qui en viennent à s'imaginer qu'ils possèdent les rues. (Pour tester cette proposition, il faut bien sûr contrôler la peur de l'agression dans l'obscurité ; je discuterai ce point plus loin dans les septième et huitième parties). De même, une proportion importante des gens qui sont dehors la nuit cherche à s'extraire des contraintes sociales, voire de la persécution. Les gens à la rue et les homosexuels, par exemple, voient dans la nuit un havre de paix du fait d'une moindre surveillance. Certains noctambules sont des ermites urbains. Certains individus qui sont embêtés ou stigmatisés - comme ceux qui sont particulièrement laids ou obèses - évitent le jour pour ne pas être ennuyés ou humiliés. Ils restent éveillés plus tard, sortent quand la plupart de leurs concitoyens sont rentrés, et se sentent plus en sécurité quand ils fréquentent les marchands de journaux, les portiers et les concierges de nuit. En d'autres termes, la nuit offre un exutoire. Comme l'Ouest, la nuit remplit une fonction d'isolement qui prévient les tensions potentielles associées aux rencontres non souhaitées.

\section{L'isolement des nouvelles colonies}

Les migrations au-delà du périmètre d'activité de la société sont d'abord éparses. Les colonies du Far West étaient petites et éloignées les unes des autres. Il y avait peu de communication entre les différentes zones et beaucoup se sont développées en autarcie. Les gens de l'Est ne voyaient pas l'intérêt des activités liées à la conquête de l'Ouest pour leur propre existence et les pionniers étaient indifférents à la société extérieure ${ }^{23}$.

À mesure que se déroulent les différents moments d'une journée, la ville passe d'actions coordonnées à des actions non coordonnées. Les zones d'activités sont éloignées les unes des autres, et elles sont plus réduites la nuit que le jour ; il y a aussi moins de communications entre ces différentes zones. Les gens du jour accordent peu d'intérêt à ceux qui sont actifs la nuit et ne les considèrent pas comme faisant partie de la même communauté.

\section{Une administration du territoire d'abord décentralisée}

Quelles que soient les élites qui décident des lois et des politiques dans une nation ou une communauté, certains administrés se situent hors de portée des dirigeants nationaux et prennent des décisions qui sont normalement de la compétence de ces derniers ou, du moins, dépendent de leur approbation. À mesure que la frontière a été repoussée et s'est éloignée du centre politique de

23. Billington R. A., op. cit., p. 96 et 746 . 
la nation, l'interprétation de la loi et des décisions judiciaires a été faite par des individus moins contrôlés consultant rarement leurs supérieurs hiérarchiques. Hollon souligne que la justice était souvent rendue « loin des tribunaux... [et] que les pionniers décidaient de manière discrétionnaire d'appliquer certaines lois et d'en ignorer d'autres, quand ils n'appliquaient pas purement et simplement leur propre loi 24 ».

Aujourd'hui, bien que de nombreuses villes et organisations soient actives en continu, leurs dirigeants de premier plan - directeurs, chefs de service, maires - sont généralement en service uniquement le jour. Quand ils vont se coucher, une semblable décentralisation des pouvoirs s'ensuit. Dans une certaine mesure, il s'agit d'une délégation de pouvoirs tout à fait explicite. Mais cette autonomisation s'explique aussi par d'autres raisons. Ainsi, les infirmières de nuit ne réveillent pas le médecin de garde car celui-ci pourrait s'agacer d'être continuellement dérangé pour des problèmes mineurs ${ }^{25}$. Le chef d'équipe fait de même avec le directeur d'usine. Des fonctionnaires moins gradés prennent des décisions qui, pendant la journée, reviennent aux cadres les plus hauts placés. Les modalités selon lesquelles une organisation ou une ville est gérée peuvent de ce fait être différentes, de même que le contenu des décisions prises. Par exemple, pour des cas similaires, les décisions prises par des officiers de police la nuit s'appuient sur des considérations moins professionnelles et plus personnelles. Ainsi, les décisions pourront être strictes ou laxistes, abusives ou humaines.

\section{L'émergence de nouveaux styles de comportement}

La conquête du territoire comme la conquête de la nuit génère plus d'individualisme car les nouveaux espaces sont éloignés et l'environnement est inhabituel (comparé aux centres de la société), et ceux qui éprouvent ces conditions relativement inédites font preuve de tolérance. Ceux qui sont partis vers les frontières de l'Ouest avaient rompu avec la société ordinaire. Le contrôle social, l'autorité établie, les lieux familiers et les normes qui y étaient associées n'avaient en effet plus cours. Ceci rend possible des comportements inhabituels. Les gens de la côte Est pensaient que «ceux de l'Ouest » étaient peu délicats. Un président de l'université de Yale pouvait ainsi affirmer : « La classe des pionniers ne peut pas vivre dans la société ordinaire. Ils sont trop oisifs, trop bavards, trop passionnés, trop prodigues, trop paresseux pour acquérir des propriétés ou du tempérament $26 »$. Un autre voyageur de la même époque pouvait écrire : «il est vrai qu'il y a des gens inutiles ici [dans des colonies situées à des centaines de miles de tout tribunal] et la plupart, il

24. Hollon W. E., « Frontier violence: another look », in R. A. Billington (ed.), People of the Plains and Mountains, Westport, Ct., Greenwood Press, 1973, p. 96.

25. Kozak L. J., « Night people: a study of the social experiences of night workers », Summation, $\mathrm{n}^{\circ} 4$, 1974, p. 59.

26. Cité dans Turner F. J., op. cit., p. 251. 
faut l'avouer, viennent de Nouvelle-Angleterre 27 ». Il poursuivait en reconnaissant quand même qu'il y avait aussi beaucoup de gens de valeur.

La déviance a aussi été le produit de la vie dans l'Ouest. Beaucoup de femmes de pionniers ont vécu dans les plaines pendant de longues périodes, sans relations sociales ordinaires, en particulier lorsque leurs maris partaient en expédition pendant des jours, voire des semaines. Ces femmes sont souvent devenues introverties et taciturnes, si timides et mal à l'aise devant des inconnus qu'elles s'enfuyaient lorsque quelqu'un s'approchait ${ }^{28}$. D'après les témoignages dont nous disposons, ces femmes étaient heureuses et tout à fait normales dans les villes où elles avaient grandi, mais elles ont été très affectées par l'environnement particulier dans lequel elles ont ensuite été amenées à vivre. Dans l'Ouest, les gens étaient habitués à ce type de comportement de la part de femmes isolées et solitaires, et ils l'acceptaient. Dans les villes de l'Est, le même comportement aurait été considéré comme tout à fait étrange.

Ceci fait là aussi écho à une image courante de la nuit comme repère d'individus bizarres et de personnages étranges, comme l'illustrent des commentaires du type : "Je ne sais pas où ils se cachent pendant la journée mais ce qui est sûr, c'est qu'ils sortent après la tombée de la nuit ». De plus, on trouve la nuit des personnes qui ont vécu des vies tout à fait normales mais qui ont été exposées à des circonstances exceptionnelles qui les ont amenées à des comportements inhabituels. Becker en rend bien compte à propos des musiciens de jazz ${ }^{29}$. Ils travaillent tard le soir et ont de ce fait peu l'occasion de fréquenter des « hommes du jour » dans leurs sorties après minuit. Le milieu est porteur d'une subculture déviante qui est tolérée et même attendue.

\section{Plus de désordre et de violence}

Les confins de l'Ouest comme la nuit ont la réputation d'être des espaces dangereux et des zones hors la loi. Il faut souligner ici qu'ils ne sont pas à la hauteur des mythes auxquels ils sont associés, dans la mesure où les agressions sont à la fois sélectives et localisées.

D'un côté, il y a des preuves évidentes de désordre et de violence. [L'historien] Walter P. Webb soulignait que l'Ouest était une zone de non droit « parce que la loi qui était censée s’y appliquer n'était pas adaptée à la situation [...], elle ne correspondait pas aux besoins du pays et ne pouvait donc pas être respectée $30 \%$. Il y avait aussi un manque de policiers et les

27. Flint T., «Frontier society in the Mississippi valley », in Ridge M. et Billington R. A. (eds.), America's Frontier Story, New York, Holt, 1969 [1826], p. 402.

28. Humphrey S. K, Following the Prairie Frontier, Minneapolis, University of Minnesota Press, 1931, p. 128.

29. Becker H. S., Outsiders: Studies in the Sociology of Deviance, New York, Free Press, 1963, p. 79,97 et 98 . 
organes de répression étaient peu nombreux ${ }^{31}$. Il y avait en particulier beaucoup de violence dans les champs aurifères ${ }^{32}$. Dans les villes de cowboys, les camps d'exploitation minière et les villes champignons des débuts de la conquête de l'Ouest, tout le monde ou presque portait une arme à feu. Le vocabulaire du combat, le bruit des révolvers, les gémissements de douleur constituaient le paysage sonore habituel. Certaines colonies de l'Ouest étaient connues pour leur concentration de joueurs, d'escrocs et de bandits, leurs danseuses, leurs bars musicaux (les «bonky-tonks») et leurs maisons de débauche. Le vol de chevaux était très répandu. Les diligences étaient régulièrement braquées. Il y avait toujours la crainte d'une attaque d'indiens ou de rebelles. Face à cela, les gens étaient constamment sur leur garde et se regroupaient pour se protéger ${ }^{33}$, d'où l'existence de groupes d'auto-défense dans les villes. Quant aux chariots couverts qui traversaient les plaines, ils étaient escortés par des cowboys armés.

Cependant, la violence était concentrée dans certains endroits ; ailleurs, les meurtres et la vindicte populaire étaient tout à fait rares. Les villes tristement célèbres de Tombstone et Deadwood, de même que les États du Texas et de Californie, avaient plus que leur part de combats armés ${ }^{34}$. Mais l'agitation dans les villes de l'Ouest était saisonnière et avait lieu lorsque les cowboys atteignaient enfin Abilene, Ellsworth et Dodge City après un long périple. Et le chaos était sélectif. Comme l'écrivait [à l'époque] Timothy Flint : "des meurtres, à la fois nombreux et horribles, ont eu lieu dans mon voisinage $[\ldots]$; ils avaient en commun l'ivresse, la brutalité et la violence [...]. [Pourtant] les hommes calmes et sobres ne courraient aucun risque d'être impliqués dans ces évènements 35 ». Même constat chez W.T. Jackson : " les homicides et les meurtres arrivaient si peu souvent que lorsque cela se produisait la communauté était choquée et scandalisée 36 ». À propos de cette violence, Hollon explique combien il y avait :

« une tendance naturelle à exagérer la vérité et à survaloriser l'exception [...]. Pas un seul duel armé, tel qu'on en a vu des milliers de fois à la télévision, n'a eu lieu dans la rue principale de Dodge City ni dans toute autre ville de cowboys du Kansas 37 ».

30. Cité par Frantz J. B. et Choate J. E., The American Cowboy: The Myth and Reality, Norman, University of Oklahoma Press, 1955, p. 83.

31. Riegel R. E., op. cit., p. 627 ; Billington R. A., op. cit., p. 480.

32. Hollon W. E., Frontier Violence, New York, Oxford University Press, 1974, p. 211.

33. Billington R. A., The American Frontiersman, London, Oxford University Press, 1954, p. 8 ; Doddridge J., "Life in the old west ", in Ridge M. et Billington R. A. (eds.), America's Frontier Story, New York, Holt, 1969 [1912], p. 103.

34. Frantz J. B. et Choate J. E., op. cit., p. 83 ; Billington R. A., op. cit., p. 63 ; Hollon W. E., 1973, art. cit., p. 96.

35. Flint T., art. cit., p. 401.

36. Jackson W.T., «Pioneer life on the plains and in the mines », in Billington R. A. (ed.), People of the Plains and Mountains, Westport, Ct., Greenwood Press, 1973, pp. 97-98.

37. Hollon W. E., art. cit., pp. 97-98. 
Pourquoi alors cette réputation de terre sauvage associée au Far West ? L'une des raisons peut être que les meurtriers hors-la-loi se déplaçaient, si bien qu'une seule personne peut avoir réalisé ses exploits sur une zone étendue. Un autre fondement de cette réputation est l'ennui. Les histoires de violence ont perduré et se sont répandues parce qu'il y avait peu à faire et peu à lire dans les maisons de pionniers. L'ennui de la vie quotidienne était compensé par des histoires excitantes racontées et répétées autour du poêle du magasin général.

À cet égard, il est possible que les desperados du Far West et les agresseurs nocturnes développent des attitudes semblables. Tous croient qu'ils sont moins visibles, et que cela accroit leurs chances de réussite eu égard aux risques qu'ils prennent. L'un parie sur les embuscades, l'autre profite de l'obscurité pour mettre en place un guet-apens. Il est facile de se volatiliser car dans les deux cas, on quitte la scène du crime pour rejoindre des zones peu fréquentées, échappant ainsi à de potentiels poursuivants.

La vie nocturne a aussi été présentée comme le lieu du mal. Elle est considérée comme criminogène et hors du contrôle social ordinaire. Les villes du Moyen Âge et de la Renaissance n'avaient pas d'éclairage public. Les agressions par les bandits et les voleurs étaient si fréquentes après la tombée de la nuit que les voyageurs se sont mis à en payer d'autres qui les précédaient en portant des torches allumées. Au XVII siècle cette escorte était appelée «link boy » à Londres et « falot » (le compagnon à la lanterne) à Paris. La fourniture de marchandises au marché noir, comme par exemple l'essence aux stationsservices pendant l'embargo sur le pétrole de 1973-1974, a été faite sous couvert de l'obscurité. Être hors-la-loi est alors possible dans la mesure où la surveillance policière est sporadique ${ }^{38}$. De plus, les policiers en service sont souvent peu disponibles car ils dorment dans leur voiture, une vieille tradition à New-York, appelée « cooping ». La même pratique m’a été rapportée informellement à propos des policiers de Boston : les joggers du petit matin les trouvent régulièrement en train de roupiller dans leur voiture à l'Arboretum.

À Boston aujourd'hui, il est plus fréquent de porter une arme la nuit. Par crainte des agressions ou du viol, des services d'accompagnement sont proposés dans beaucoup de campus pour les femmes qui rentrent la nuit, ou pour les femmes qui travaillent le soir et quittent leur lieu de travail pour rejoindre le parking ou le métro. Une escorte est également proposée aux infirmières de l'hôpital de Boston du fait de la recrudescence des vols. Et certaines résidences, dont l'entrée est gardée par un vigile, ont été transformées en forteresses verticales vers lesquelles les gens se replient la nuit.

38. «Boston police today ", Boston Globe, 4 avril 1977, p. 1. 
Cependant, comme autrefois dans le Far West, le désordre et la violence nocturnes concernent certaines heures et se concentrent sur certains lieux, tandis qu'ils sont rares ailleurs. Les bagarres atteignent un pic vers minuit, comme le montre le graphique $\mathrm{n}^{\circ} 3$, mais elles sont moins fréquentes entre $2 \mathrm{~h} 30$ et 11 heures du matin. Le quartier de Boston où il y a le plus d'agressions et de rixes, où la prostitution est forte, et où se situent les bars et salons où se produisent des «gogo dancers » nus, est appelée « la zone de combat » («combat zone»). Une population de passage constituée d'hommes relativement jeunes vient dans le quartier pour fréquenter les cinémas pornographiques ; ils s'enivrent et deviennent agressifs, dans les bars comme dans la rue. Bien que cette description se rapproche de ce qui a jadis été dit des villes minières de l'Ouest, ces « zones de combat » ne fonctionnent plus vraiment après $2 \mathrm{~h} 30 \mathrm{du}$ matin ni pendant la journée. Le jour, ces zones font partie des quartiers d'affaires. Beaucoup de gens font leurs courses dans les magasins qui se trouvent à proximité, ou bien traversent la zone et en fréquentent les restaurants et les entreprises. Cette dénomination de «zone de combat » s'applique ainsi à ces endroits seulement à certaines heures et n'est pas valable pour toute la ville toute la nuit.

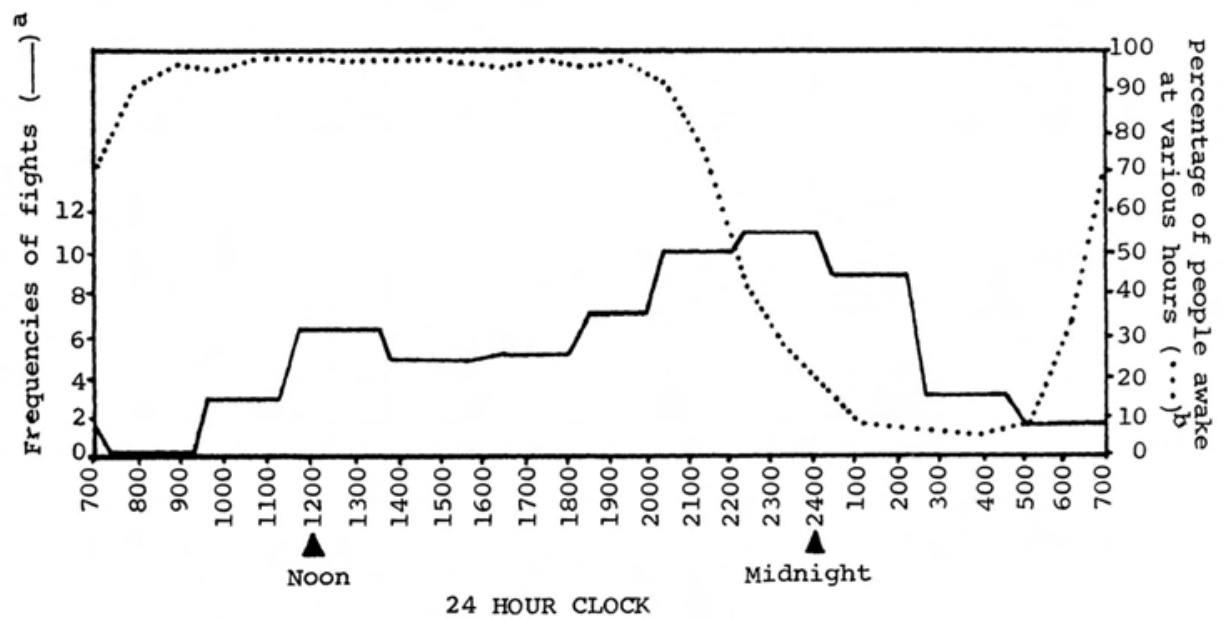

Graphique 3. Nombre de bagarres déclarées et pourcentage de personnes éveillées dans la ville de Boston

Bagarres signalées via des appels d'urgence dans la ville de Boston, sur la base d'un échantillon de 49 jours (24h/24) en 1974 (juin, septembre et décembre). Les fréquences sont lissées du fait des variations dans l'échantillon sur 24 heures.

Le pourcentage de gens éveillés est une estimation pour Boston et s'appuie sur des données relatives à 44 villes américaines, citées dans Szalai A., op. cit., p. 737. 
À l'époque de la conquête de l'Ouest, Hollon note que " pour chaque acte de violence, il y avait des milliers d'exemples de gentillesse, de générosité et de sacrifice... 39 ». Il cite un voyageur anglais qui observait que "les frustes hommes de l'Ouest, les fils rustiques de la lutte contre les Indiens, habitués depuis l'enfance à lutter pour leur survie, étaient accueillants et généreux à un degré difficilement égalable dans la société plus civilisée ».

Les récits sur la vie au Far West évoquent souvent la chaleur manifestée envers les étrangers, la construction collective des maisons, l'édification de granges et l'attention aux personnes dans le besoin ${ }^{40}$. « Les voisins étaient disposés à prêter tout ce qu'ils possédaient. Aucun homme conduisant un chariot vide sur une bonne route ne serait passé devant un homme à pieds sans l'inviter à monter dans son véhicule 41 ». Les voyageurs revenant des contrées périphériques racontaient qu'ils étaient traités avec plus d'égards qu'ils ne l'avaient été dans les villes ${ }^{42}$.

De prime abord, ces histoires sur l'hospitalité généreuse des gens de l'Ouest peuvent sembler en décalage avec les forts risques de vol et de violence. Mais ces deux phénomènes sont en fait liés. Dick soulignait que « tandis que les colons isolés se battaient contre les sauvages [...] et la solitude, ils étaient unis par une forme de camaraderie 43 ». Pour Billington :

« La coopération est normale à l'intérieur d'un groupe, mais elle devient plus forte lorsque le groupe est en conflit avec un groupe extérieur ; la solidarité du groupe s'accroît. Telle était la situation dans les communautés du Far West, où les conflits avec les Indiens, avec la nature sauvage et avec les gens de l'Est qui les dominaient renforçaient l'esprit de solidarité 44 ».

Schnachter a démontré de manière expérimentale que, dans de telles conditions, les gens cherchent à s'associer à leurs semblables 45 . Il a mis en évidence que plus les gens avaient le sentiment d'être confrontés à un grand danger, plus ils étaient inquiets; et plus ils étaient inquiets, plus ils voulaient être

39. Hollon W. E., op. cit., pp. 211-212.

40. Darby W., "Primitivism in the lower Mississippi valley », in Ridge M. et Billington R. A. (eds.), America's Frontier Story, New York, Holt, 1969 [1818], p. 400 ; Frantz J. B. et Choate J. E., op. cit., p. 64 ; Billington R. A., op. cit., pp. 96, 167 ; Riegel R. E., op. cit., p. 81.

41. Dick E., op. cit., p. 512.

42. Flint T., art. cit., pp. 402-403 ; Hollon W. E., op. cit., p. 212.

43. Dick E., op. cit., p. 510.

44. Billington R. A., "Frontier democracy: social aspects », in Taylor G. R. (ed.), The Turner Thesis: Concerning the Role of the Frontier in American History, 3e éd., Lexington, Ma., Heath., 1972, p. 166.

45. Schachter S., The Psychology of Affiliation, Stanford, Stanford University Press, 1959. 
avec d'autres personnes - même des inconnus - confrontées aux mêmes dangers. Schnachter en concluait qu'être dans le même bateau que d'autres réduit l'inquiétude et permet d'évaluer ses sentiments et de les ajuster aux risques. La moindre incertitude émotionnelle et le fait de savoir que d'autres partagent la même situation, conduisent à se sentir plus apte à affronter une situation éprouvante.

Parce que la nuit est un moment de plus grande violence et que les gens se sentent alors plus vulnérables, ceux qui s'y promènent ont des attitudes semblables et se comportent les uns envers les autres de la même façon que les pionniers. La nuit, les gens sont plus attentifs aux inconnus quand ils passent dans la rue. Chacun jauge l'autre pour savoir s'il est dangereux. Quand il s'agit de décider si l'autre est digne de confiance ou pas, on passe de la vigilance à l'ouverture. Si ce n'est pas un ennemi, alors c'est un ami. Conscients d'être ensemble dans un même environnement dangereux, les gens s'identifient les uns aux autres et deviennent plus extravertis. Le sentiment de sécurité qui gagne ceux qui se retrouvent ensemble la nuit pour un dîner ou dans un café favorise la camaraderie.

Par ailleurs, dans ces deux espaces-frontières, il se peut que les gens soient plus accueillants parce qu'ils ont plus de temps à consacrer aux inconnus. Les pionniers avaient beaucoup à faire ; cependant, ils n'avaient souvent rien à faire. Ils n'étaient pas aussi contraints par les tâches quotidiennes que l'étaient les gens dans les villes de l'Est, et la ponctualité n'était pas une norme aussi valorisée. Un homme qui a grandi au Far West :

«... se souvenait de l'ennui auquel il ne pouvait échapper [...]. [L]e pire moment était le dimanche après-midi, quand il n'avait rien à faire. Il n'y avait pas de journaux à lire et aucun autre livre que la Bible familiale, il n'avait personne de son âge à qui parler et le magasin le plus proche était à des kilomètres 46 ».

En ville, le jour, les emplois du temps sont pesants, si bien que les rencontres sont des moments inhabituels et courts. Le rythme se ralentit de façon nette après minuit. Ceux qui sont dehors sont moins pressés, ils ont moins à courir pour aller à tel ou tel endroit. Tandis que le manque de temps empêche la sociabilité et l'entraide, le temps disponible les rend possibles.

J'ai vérifié la pertinence de ces idées à travers quatre tests sur l'altruisme et la convivialité à différentes heures du jour et de la nuit. Le test concerne des situations ordinaires, pas des situations d'urgence auxquelles il faut répondre dans des conditions de stress mais des situations s'inscrivant dans le flux habituel des événements sociaux. Les résultats concernant le degré d'altruisme et 
de convivialité ont été établis en demandant à une série d'individus de noter les situations (dix « juges » pour le test $n^{\circ} 3$, six pour chacun des tests $n^{\circ} 1,2$ et 4).

Test $n^{\circ} 1$ : Demander son chemin. Un couple composé d'un homme et d'une femme utilisaient une méthode d'échantillonnage aléatoire dans la rue 47 pour approcher les passants et leur demander leur chemin vers un lieu connu situé à environ un mile. C'est une question banale, qui demande peu de temps et peu d'effort, et qui n'appelle pas une réponse personnalisée. Indiquer le chemin conduisait à l'attribution d'un point. Une réaction désagréable - par exemple, s'écarter brutalement pour ignorer un mendiant, se retourner pour proférer des termes obscènes ou un abrupt « Demande à quelqu'un d'autre », se détourner rapidement de l'interlocuteur comme s'il n'était pas là, le fixer rageusement tout en continuant sa route - valait zéro point. Si la personne interpellée élargissait le cadre de la rencontre - par exemple en disant, en plus d'indiquer le chemin, «Vous êtes en vacances par ici ?» ou « Mon fils vit làbas » ou «Vous avez besoin que je vous dépose ? Ma voiture est à côté »valait 2 points. Après chaque test, les deux chercheurs de terrain s'accordaient sur la note finale. Sur 363 personnes approchées, 331 (91,2\%) ont seulement indiqué le chemin, $21(5,8 \%)$ ont donné une autre dimension à la rencontre et $11(3 \%)$ ont refusé de manière désagréable.

Test $n^{\circ} 2$ : Demander un court entretien. Un couple composé d'un homme et d'une femme suivait un protocole d'échantillonnage aléatoire dans la rue (voir note de bas de page 47) pour approcher les passants et leur demander de répondre à quelques questions dans le cadre d'une étude portant sur les citadins. C'est un type d'interaction moins commun mais qui n'est pas inhabituel

47. Les tests 1,2 et 3 évoqués dans cette section ont été menés pendant chacune des 166 visites de terrain pour lesquelles l'échantillon est décrit dans la Figure 2. Une équipe de 2 chercheurs, toujours un homme et une femme, faisaient le travail de terrain. C'était toujours l'homme qui demandait son chemin, mais les deux essayaient d'obtenir des entretiens (test 2). Les tests 1, 2 et 3 ont été menés dans des endroits éclairés la nuit, par exemple sous un lampadaire. Les tests 1 et 2 ont tous deux demandé un échantillon aléatoire de passants et la procédure utilisée a été le décompte des personnes par tranche de $5 \mathrm{mn}$, qui a fourni les données qui figurent sur le graphique 2. Le décompte a permis d'établir la densité de population dans la rue à l'heure donnée, et la densité a été utilisée pour déterminer le taux d'échantillonnage : si le nombre était supérieur à 25, la quatrième personne venant de la direction choisie après le lancement du test était sélectionnée ; si le nombre allait de 5 à 25 , la troisième personne était choisie ; si trois ou quatre personnes passaient toutes les 5 minutes, la deuxième personne était ciblée ; et si deux ou moins de deux personnes passaient toutes les 5 minutes, alors la première personne venant de l'une ou l'autre direction était retenue. Les membres d'un même groupe de personnes étaient décomptés comme une seule personne ; si le groupe était retenu du fait du mode d'échantillonnage, c'est le passant le plus près du chercheur qui était retenu. Un maximum de 3 demandes de direction et de 8 demandes d'interview pouvait être réalisé à chaque sortie de terrain. Parfois, les tentatives étaient moins nombreuses, notamment la nuit, parce que presque personne ne passait pendant le temps prévu pour le travail de terrain. L'échantillonnage était réalisé de manière silencieuse par l'un des chercheurs, qui signalait à l'autre qui aborder, par un coup de coude ou tout autre signe discret. Ceci minimisait les risques de biais de sélection : le chercheur qui devait aller faire le test ne pouvait pas hésiter ni ignorer un passant peu attrayant. 
dans ce pays où les sondages sont fréquents. On demande alors d'accorder du temps et de la confiance à des inconnus : le questionnaire prend en effet plusieurs minutes et les questions sont personnelles - elles concernent les sentiments, le travail, les conditions de vie. Un refus désagréable - par exemple un " Je ne veux pas [répondre à cette enquête] » sèchement prononcé ou l'une des réactions désagréables listées ci-dessus pour le test 1 - valait zéro. Un refus poli - comme une réponse du type «Je suis désolé, j’ai un rendez-vous » ou une formule de politesse équivalente - se voyait attribuer 1 point. L'acceptation valait 2 points. Sur 1129 tentatives, 175 (15,5\%) ont refusé de manière désagréable, 258 (22,9\%) ont refusé gentiment et 696 (61,6 \%) ont accepté de répondre au questionnaire.

Test $n^{\circ} 3$ : Trouver une clé perdue. Une clé trouvée dans la rue est susceptible d'être considérée comme un objet de valeur appartenant à quelqu'un que la personne qui a découvert la clé ne connaît pas. Cette dernière se soucie-telle assez d'un inconnu qui a perdu sa clé pour la lui renvoyer ? C'est une situation d'anonymat. Il n'est pas nécessaire de rentrer dans une relation personnelle avec le propriétaire de la clé pour la lui redonner. Ce test vise à vérifier s'il y a une différence selon l'heure dans les taux de retour des clés parmi ceux qui les ramassent et les emportent. L'idée d'utiliser une clé pour une telle expérience a été développée par Forbes, Te Vault et Gromoll ${ }^{48}$. Au début de chaque sortie de terrain (voir note de bas de page 47), les chercheurs plaçaient des clés en aluminium brillant et coloré dans des endroits bien éclairés de la rue. Chaque clé avait une étiquette indiquant le nom et l'adresse de quelqu'un dans une ville située à plus de 90 miles (Northampton) ainsi que la mention «À renvoyer, s'il vous plait ». Nous avons évité les endroits proches d'entrées de magasin dans la mesure où les clés auraient alors pu être remises à l'employé plutôt qu'envoyées par la poste. Les clés respectaient un code couleur et avaient été entaillées, afin que nous puissions relier chaque clé renvoyée à un intervalle de temps particulier sur le cycle de 24 heures. À la fin de chaque sortie de 2 heures, les chercheurs faisaient un tour pour reprendre les clés qui n'avaient pas été ramassées.

Au total, sur 326 clés ramassées, 220 (67,5\%) ont été renvoyées. Si la clé était déposée dans la boîte aux lettres (à partir de laquelle elle était adressée à Northampton, où les frais postaux étaient payés), un point était attribué ; 154 clés $(47,5 \%)$ ont été renvoyées de cette façon. Les 38 clés (11,7\%) qui ont été renvoyées dans une enveloppe timbrée ont eu 2 points chacune. 25 autres clés, emballées et timbrées (7,7\%), étaient accompagnées d'un mot personnel et ont obtenu 3 points chacune. Trois individus $(0,9 \%)$ ont même appelé à Northampton pour dire que la clé était entre de bonnes mains, et ont été cré-

48. Forbes G. B., Te Vault R. D. et Gromoll H. F., «Regional differences in willingness to help strangers: a field experiment with a new unobtrusive measure ", Social Science Research, vol. 1, n²4, 1972, pp. 415-419. 
dités de 4 points chacun. Les différentes réactions renvoient à des degrés accrus d'investissement et de temps consacré pour aider un inconnu. Les 106 clés $(32,5 \%)$ qui n’ont pas été renvoyées ont obtenu zéro point.

Test $n^{\circ} 4$ : Être sociable dans un supermarché. La caisse de supermarché est un modèle réduit de la vie urbaine. Les gens, qui sont pour l'essentiel des inconnus les uns pour les autres, sont ici en contact dans une situation brève et balisée. Les assistants de recherche, des couples homme/femme, suivaient une procédure d'échantillonnage aléatoire stratifié ${ }^{49}$ pour aller dans 3 supermarchés ouverts $24 \mathrm{~h} / 24$ dans différentes parties de Boston à différentes heures du jour et de la nuit. Ils se faisaient passer pour des clients et notaient le degré de sociabilité entre les clients et les employés aux caisses. La sociabilité est définie comme "le fait de manifester de la chaleur et d'étendre la portée d'une interaction avec quelqu'un d'autre ». Dans ce cas, le sourire était considéré comme une marque de chaleur. Le critère d'élargissement de la portée d'une interaction était de discuter sur un sujet autre que la transaction - comme dire à l'employé «vous êtes étudiant ? » ou «vous avez une belle chemise, où l'avez-vous trouvée » ou parler de l'actualité. Si le client souriait et discutait, la rencontre se voyait attribuer deux points. Si seul l'un des deux phénomènes se produisait, on ne donnait qu'un point, et zéro si rien ne se passait. Les interactions entre les clients et les employés ont révélé un haut degré de réciprocité. Lorsque l'un était impassible, l'autre l'était aussi, et lorsque l'un était sociable, l'autre répondait de la même façon. C'est pourquoi ce schéma de notation simple a été utilisé plutôt qu'un décompte qui aurait inclus le comportement des deux parties. Dans quelle mesure les gens dépassent-ils les limites de leur rôle à la caisse du supermarché pour offrir ne serait-ce qu'un peu de sociabilité ? Cela ne va pas loin : $562(74,7 \%)$ des 752 clients n'ont pas souri ni parlé de quoi que ce soit d'autre que la transaction. Le fait de sourire a été observé 98 fois (13\%), et seulement $66(8,8 \%)$ ont à la fois discuté et souri.

Pour résumer, environ 2500 personnes ont été observées dans différentes parties du centre-ville de Boston tout au long d'une journée de 24 heures et ont été évaluées sur la manière dont elles ont réagi à quatre situations : donner des directions à ceux qui les ont interrogées ; accepter d'être interviewées ;

49. L'échantillon du supermarché était semblable à celui des expériences réalisées dans les rues de Boston (voir figure 2). Les mêmes intervalles de temps étaient notés dans le schéma d'échantillonnage et trente visites ont été sélectionnées aléatoirement (trois heures pendant la période de forte fréquentation du matin ; sept pendant la journée, quatre pendant la période de forte fréquentation du soir, sept le soir et neuf la nuit). Le quota pour les visites de nuit était plus élevé parce que moins de clients étaient observés à chaque fois. Les observateurs étaient 11 binômes d'étudiants de mon cours d'Interaction sociale de l'automne 1973 ; ils ont été formés et se sont entraînés à travers des essais antérieurs jusqu'à ce que l'indice de confiance atteigne 0,92 (au sein des binômes d'observateurs). Un des supermarchés se situe à 500 mètres de la zone résidentielle où ont été menées les expériences de rue; les deux autres sont situés dans différentes parties de la ville. À chaque sortie de terrain, les observateurs suivaient un ordre de passage toujours différent dans les trois supermarchés, pour un total de 90 observations de 15 minutes. 
retourner les clés perdues qu'elles ont trouvées; et être sociable avec des inconnus au moment de payer ses courses à la caisse d'un supermarché.

Quatre tests ont été mis en place afin que plusieurs comportements différents puissent définir et valider ce qui est étudié. S'ils ne couvrent pas l'ensemble des attitudes serviables et amicales, manifester de la convivialité, coopérer avec quelqu'un d'apparence modeste et étendre la portée d'une interaction sont au cœur de telles relations.

Selon les tests, les échantillons d'individus ne sont pas les mêmes. Les tests 1 et 2 utilisent une sélection régulière de passants suivant une procédure aléatoire ajustée à la densité de population dans la rue. Le test 3 se concentre seulement sur les personnes qui ramassent les clés. Le test 4 concerne uniquement des consommateurs seuls à la caisse des supermarchés ouverts 24h/24. Cependant, les comparaisons en temps réel sont appropriées, puisque les tests sont tous fondés sur des plans d'échantillonnages aléatoires qui se sont déroulés à la même fréquence tout au long du cycle de 24 heures. Pour tester les hypothèses, il s'agira de mesurer les écarts selon les moments du jour pour chacun des tests et d'observer la cohérence des résultats à chaque heure entre les quatre tests.

Le tableau 2 rend compte des résultats des tests. On observe une impressionnante régularité pour trois d'entre eux, dont les scores les plus élevés sont ceux mesurés pendant la nuit. Non seulement la période nocturne offre les résultats les plus élevés dans ces trois cas, mais on constate aussi qu'aucun autre moment dans la journée n'apparaît de façon systématique en seconde position. Dans certains cas, les divergences entre la nuit et le créneau horaire dont les caractéristiques sont les plus proches ne sont pas statistiquement significatives, même si l'analyse de la variance donne des résultats significatifs lorsque toutes les séquences sont comparées. Bien que les écarts selon les heures soient faibles dans certains cas, l'effet cumulatif de ces pratiques est susceptible de faire une nette différence dans l'atmosphère propre à telle ou telle période de la journée.

La tendance générale confirme l'hypothèse que la nuit est une période de plus grande serviabilité et cordialité que les autres séquences de la journée. De ce point de vue, le résultat du test des clés est étonnant. La nuit a de loin le plus mauvais taux de serviabilité. Elle correspond à la plus faible proportion de clés retournées $(50 \%)$ et c'est là qu'on observe le moins d'effort supplémentaire, au-delà de l'action de déposer les clés non emballées dans une boite aux lettres. La conclusion est si nette et si contraire aux attentes qu'elle doit être significative. Son interprétation pourra profiter d'informations qu'il reste à présenter, et je proposerai plus tard des commentaires quant à son rapport avec l'hypothèse du front pionnier. 


\section{Période de la journée}

\begin{tabular}{|c|c|c|c|c|c|c|c|}
\hline \multirow[b]{2}{*}{ Test } & \multicolumn{5}{|c|}{ Période de la journée } & \multirow[b]{2}{*}{$\Sigma$} & \multirow{2}{*}{$\begin{array}{l}\text { Analyse } \\
\text { de la } \\
\text { variance } \\
\text { et signifi- } \\
\text { cativité }\end{array}$} \\
\hline & $\begin{array}{c}\text { Heure } \\
\text { de pointe } \\
\text { du matin } \\
(7: 30-9: 29)\end{array}$ & $\begin{array}{c}\text { Jour } \\
(9: 30-16: 14)\end{array}$ & $\begin{array}{c}\text { Heure } \\
\text { de pointe } \\
\text { du soir } \\
(16: 15-18: 15)\end{array}$ & $\begin{array}{c}\text { Soir } \\
(18: 15-00: 14)\end{array}$ & Nuit & & \\
\hline \multirow{2}{*}{$\begin{array}{l}\text { 1. Indiquer } \\
\text { le chemin a }\end{array}$} & $\mathrm{n}=32^{\mathrm{e}}$ & $n=115$ & $n=27$ & $\mathrm{n}=123$ & $n=66$ & $\mathrm{n}=363$ & $F=4,917$ \\
\hline & 1,06 & 0,97 & 1,07 & 1,00 & {$[1,15]^{\mathrm{e}}$} & 1,03 & $p<0,001$ \\
\hline \multirow{2}{*}{$\begin{array}{l}\text { 2. Accepter } \\
\text { d'être inter- } \\
\text { viewéb }\end{array}$} & $n=93$ & $n=366$ & $n=81$ & $n=363$ & $\mathrm{n}=226$ & $\mathrm{n}=1129$ & $F=4,531$ \\
\hline & 1,17 & 1,45 & 1,46 & 1,50 & {$[1,55]$} & 1,46 & $p<0,002$ \\
\hline \multirow{2}{*}{$\begin{array}{c}3 . \\
\text { Retourner } \\
\text { des clés } \\
\text { perdues c }\end{array}$} & $n=29$ & $\mathrm{n}=113$ & $n=26$ & $\mathrm{n}=94$ & $\mathrm{n}=64$ & $\mathrm{n}=326$ & $F=3,972$ \\
\hline & 0,93 & 1,00 & 1,04 & {$[1,18]$} & $0,61(!)$ & 0,97 & $p<0,01$ \\
\hline \multirow{2}{*}{$\begin{array}{c}\text { 4. Être } \\
\text { sociable } \\
\text { avec des } \\
\text { étrangers d }\end{array}$} & $n=68$ & $n=212$ & $\mathrm{n}=161$ & $\mathrm{n}=179$ & $\mathrm{n}=132$ & $\mathrm{n}=752$ & $F=5,250$ \\
\hline & 0,24 & 0,42 & 0,29 & 0,22 & {$[0,50]$} & 0,33 & $\mathrm{p}<0,001$ \\
\hline
\end{tabular}

\section{Tableau 2. Données agrégées de quatre tests distincts de serviabilité et de convivialité au cours de différentes périodes du cycle de 24 heures à Boston en 1974}

a Indicateur construit en attribuant zéro point pour des réponses désagréables et aucune direction donnée, un point lorsque seule la direction est communiquée, deux points lorsque la direction est donnée et que l'interaction ne s'y réduit pas; les données sont agrégées et divisées par le nombre d'essais (les personnes approchées) au cours de la période de temps donnée. Deux des six enquêteurs se sont distingués en notant un refus courtois - i.e. " je suis désolé, je ne sais pas »- expliquant qu'il s'agissait d'une manière banale d'éviter de s'engager. Par conséquent, les refus polis ont été exclus de l'analyse. Une vérification a pu montrer que cela ne faisait pas de différence. Il y a eu trente occurrences de refus polis, réparties sur les différentes périodes de la journée, et leur inclusion (un point pour chacun) a généré le même résultat ANOVA [analyse de variance] que leur exclusion.

b Indicateur construit en attribuant zéro point pour un refus désagréable, un point pour un refus poli, deux points pour une acceptation; les données sont agrégées et divisées par le nombre d'essais (les personnes approchées) au cours de la période de temps donnée.

c Indicateur construit en attribuant zéro point si les clés ne sont pas retournées, un point si elles sont postées telles quelles, deux points si elles sont postées dans une enveloppe, trois points si elles sont retournées enveloppées et accompagnées d'un message, quatre points si un contact personnel est engagé par téléphone ; les données sont agrégées et divisées par le nombre d'essais (les clés emportées) au cours de la période de temps donnée.

$\mathrm{d}$ Indicateur construit en attribuant un point pour un simple sourire, un point pour une discussion, deux points pour les deux, zéro point pour aucun des deux ; les données sont agrégées et divisées par le nombre d'essais (les transactions observées à la caisse) au cours de la période de temps donnée.

e $\mathrm{n}=$ nombre d'essais ; [] = score moyen le plus élevé pour le test. 
La structure des résultats des quatre tests permet de rejeter une hypothèse concurrente, selon laquelle la peur détermine la conduite des personnes à l'égard de ceux qui leur sont étrangers pendant la nuit. Nous savons que la nuit est perçue comme un moment trop dangereux pour être hors de chez soi en ville (U.S. Office of Management and the Budget, 1974, pp. 58-59 et 73). Si la crainte d'une agression criminelle dominait effectivement les comportements individuels, elle serait plus forte dans les rencontres en face-à-face qu'à l'égard d'une invitation passive et anonyme à ramasser une clé où est marquée «Ầ renvoyer s'il vous plaît ». Nous attendrions des gens qu'ils soient davantage sur leur garde face aux autres la nuit, qu'ils évitent d'être approchées par des étrangers, mais à l'inverse qu'ils soient plus serviables dans la situation faiblement risquée de mettre une clé perdue dans une boite aux lettres. Le tableau 2 nous dit que c'est pourtant le contraire qui se déroule. Les noctambules sont plus serviables et chaleureux à l'égard des étrangers en face-à-face. Et pourtant, ils renvoient moins souvent les clés ramassées par terre.

\section{L'exploitation des ressources fondamentales comme politique nationale}

L'expansion vers l'Ouest a démarré bien avant que quelqu'un ne reconnaisse officiellement les opportunités offertes par ces territoires pour notre société. Il a même fallu des années pour réaliser que l'Ouest des États-Unis pouvait être habitable. Autrefois, les terres à l'ouest du Missouri étaient décrites sur les cartes comme le Grand Désert américain. Presque personne ne pensait qu'un jour de nombreux individus souhaiteraient y migrer et s'y installer ${ }^{50}$. De même, l'expression-clé de la « Destinée manifeste » a été appliquée à la colonisation de l'Ouest à partir de 1845, des siècles après les premières initiatives. En 1837, Horace Greeley ${ }^{51}$ lançait le slogan « Go West Young Man, go forth into the Country ». Il considérait une telle migration comme un moyen de réduire la pauvreté et le chômage provoqués par la Panique de 1837. En 1854, Greeley exhortait : «Transformez les terrains libres en quarts de section ${ }^{52}$ pour les colons d'aujourd'hui [...] et les millions qui n'ont actuellement pas de terre ne seront jamais plus orphelins et mendiants 53 ». En 1862, avec le vote de la Loi de Propriété fermière, cela devint une politique explicite du gouvernement états-unien d'utiliser les territoires de l'Ouest pour soulager les conditions des exploitants agricoles et des ouvriers urbains sous pression. Pour défendre la Loi de Propriété fermière, un membre du Congrès déclarait ainsi : «Je soutiens cette mesure [...] parce que cette opération bienveillante va repousser pendant des siècles, sinon pour toujours, tout conflit sérieux entre le capital et le travail dans les plus vieux États libres ${ }^{54}$ ». Les décideurs

50. Hicks J. D., The Federal Union, Boston, Houghton Mifflin, 1948, p. 508.

51. NDT : Ouvriériste, engagé dans le combat abolitionniste, il est l'éditeur du principal journal Whig de New York (le New York Tribune), et compte parmi les membres fondateurs du Parti Républicain en 1854.

52. NDT : un quarter-section désigne une unité de mesure de 64 ha.

53. Cité par Smith H. N., Virgin Land, New York, Vintage, 1957 [1950], pp. 234-5. 
voyaient finalement dans l'exploitation des terres de l'Ouest un moyen de résoudre les problèmes sociaux.

De la même façon, pendant les 150 années qui ont suivi l'introduction de l'éclairage au gaz de houille de Murdock, il n'y a eu aucun projet national de colonisation de la vie nocturne en Angleterre ou aux États-Unis. Les gens sont allés de l'avant, étendant leurs activités aux heures sombres, sans avoir conscience qu'une communauté de 24 heures se mettait en place. Maintenant, dans les années 1970, les décideurs commencent à s’intéresser aux heures rentables de la nuit de la même façon qu'ils s'intéressaient autrefois aux terres bon marché de l'Ouest. Le soviétique V.D. Patrushev analyse ainsi que « le temps [...] est une forme particulière de richesse nationale. Par conséquent il est fondamental de prévoir son utilisation la plus efficace pour chaque membre de la société 55 ». Daniel Schydlowsky, un économiste spécialisé dans le développement en Amérique latine qui y a récemment mené une étude pendant trois ans, a conclu qu'un travail en roulement produirait des bénéfices notables en termes de réduction du chômage et profiterait à l'économie des villes en développement, qui sont surpeuplées ${ }^{56}$. Son appel à utiliser le temps rappelle l'attitude de ceux qui, au XIX ${ }^{\mathrm{e}}$ siècle, défendaient l'exploitation des terres de l'Ouest comme solution pour ceux qui n'avaient pas de travail.

Les partisans de l'expansion vers l'Ouest la voyaient également comme une façon de faire sortir un grand nombre de personnes des villes et d'éviter la surpopulation ${ }^{57}$. Aujourd'hui, George Dantzig et Thomas Saaty recommandent d'étendre les temps d'activités afin de réduire la congestion ${ }^{58}$. Meier ajoute : «Les terres, qui sont rares, et le temps des humains, qui est cher, peuvent aussi être préservés en encourageant les opérations 24h/24. Comme cela, les gens peuvent vivre densément sans se marcher sur les pieds 59 ».

\section{L'émergence de groupes d'intérêt}

À mesure que la frontière états-unienne progressait, la population est devenue plus consciente de sa situation particulière et s'est organisée pour porter ses propres revendications. Turner a noté que l'Ouest a développé une réelle conscience de ses différences avec l'Est 60. Il écrivait : «[...] Est et Ouest ont cherché à se distinguer depuis le début. Ceux qui habitaient au sein des colonies faisaient peu de cas des habitants de la côte, qui en retour les mépri-

54. Ibid., p. 239.

55. Patrushev V. D, "Aggregate time-balances and their meaning for socio-economic planning », in Szalai A. (ed.), The Use of Time, La Hague, Mouton, 1972, p. 429.

56. Schydlowsky D., "Multiple shifts would produce 'revolutionary results' for Latin American economy », Boston University, Spectrum 4, 9 septembre 1976, p. 5.

57. Smith H. N., op. cit., p. 8 et p. 238.

58. Dantzig G. B. et Saaty T. L., Compact city, San Francisco, Freeman, 1973, pp. 190-193.

59. Meier R., 1976, art. cit., p. 965

60. Turner F. J., op. cit., p. 207 ; et 1969 [1965], op. cit., p. 54. 
saient. L'Ouest a fini par se découvrir une identité propre et est même devenu rétif aux injonctions de l'Est. Il rejetait l'idée qu'il était simplement une émanation des deux rivaux, Nord et Sud, une simple dépendance de l'une ou l'autre des sections de l'Est. Il a commencé à se penser comme une section en soi ${ }^{61}$. " Les sections sont des groupes d'intérêt à base géographique. Il y a un siècle, l'Ouest a engendré de tels groupes de pression mais aussi des groupements d'agriculteurs tels que le Greenback party, le National Grange et les Populistes. Le mouvement Granger, par exemple, s'est développé en réponse aux problèmes de transport rencontrés par les habitants de l'Ouest dans leur région. Il n'y avait aucune rivière ou canal important vers l'Ouest, si bien que les colons dépendaient du bon vouloir des chemins de fer. Mais les tarifs dans les nouvelles régions de l'Ouest étaient nettement plus élevés que ceux de l'Est, et ce sont les protestations contre cette disparité qui ont contribué au mouvement dans les années 187062.

La nuit isole également un groupe du cœur de la société. Un antagonisme peut se développer à mesure que les gens du jour désapprouvent les noctambules et que ces derniers s'indignent du mépris manifesté par les autres. Les personnes actives la nuit considèrent que leur style de vie diffère de celui de la société diurne, prennent conscience d'avoir une identité séparée, et se transforment en groupes d'intérêt. De nouveaux clivages dans la veine du régionalisme commencent à émerger. Cela s'est déjà produit pour deux groupes habituellement liés à la vie nocturne : les homosexuels et les prostituées. Le Front de Libération Gay est une des organisations nationales dévolues aux droits des homosexuels. Les prostituées ont également un syndicat. Elles ont opportunément adopté le nom d'une créature connue dans l'Ouest des États-Unis pour hurler la nuit : le coyote. COYOTES (Call Off Your Old Tired Ethics ${ }^{63}$ ) revendique une loi qui décriminalise leurs activités et dénonce en justice les discriminations contre les femmes qui gagnent leur vie en se prostituant ${ }^{64}$.

Une contestation effective du jour contre la nuit a déjà été engagée à Boston. L'aéroport de la ville est encadré par une zone résidentielle et ses activités nocturnes sont devenues une nuisance pour les personnes qui ne veulent pas être perturbées pendant leur nuit de sommeil. En 1976, les riverains des environs, en tant que simples citoyens et à travers deux groupes organisés Fair Share et le Comité du Massachusetts pour la réduction du bruit et de la pollution -, se sont concertés pour faire cesser les vols aériens entre 23 heures et 7 heures. Cela a conduit le monde des affaires à contre-argumenter en mettant l'accent sur l'intérêt économique d'une poursuite des vols. Le groupe pro-vie nocturne était une coalition regroupant intérêts commerciaux, com-

61. Turner F. J., op. cit., pp. 25-30.

62. Robbins R. M., op. cit., p. 271.

63. NDT : Call Off Your Old Tired Ethics peut se traduire par Abandonne Ta Vieille Morale Désuète.

64. «Prostitutes speak of pride, but they are still victims », Boston Globe, 25 juin 1976, pp. 1 et 10. 
pagnies aériennes, syndicats et employés de l'aéroport travaillant de nuit (certains d'entre eux habitant d'ailleurs juste à côté). Ce groupe expliqua que le couvre-feu aurait pour conséquence la perte de milliers d'emplois, la disparition de millions de dollars et qu'au-delà, il découragerait les investissements dans le territoire de la Nouvelle-Angleterre. Rejointe par le gouverneur, le maire et de nombreux parlementaires, la coalition a obtenu, de la part de la Massachusetts Port Authority, la pérennisation des vols nocturnes. Quelques propositions de réduction du bruit pendant la nuit ont accompagné la décision. Un mois plus tard, Eastern Airlines annonçait qu'il ajoutait un Airbus à sa flotte et augmentait son personnel à l'aéroport, "conséquence directe de la récente décision [...] de ne pas imposer de couvre-feu à [l'aéroport de] Logan ». Comme l'a indiqué un homme d'affaires, "La décision du couvrefeu a été vue comme un règlement de compte à $\mathrm{OK}$ Corral 65 ».

\section{Discussion}

Ces preuves confirment l'hypothèse de la nuit comme «frontière ». Que les noctambules soient moins disposés à renvoyer les clés qu'ils trouvent accrédite également cette idée. Les résultats du test 3 paraissent contredire l'affirmation selon laquelle les gens seraient plus serviables dans une zone nouvellement conquise mais l'expérience de la clé perdue se distingue des autres tests en ce qu'elle est la seule dans laquelle les personnes ne se rencontrent pas en face-à-face. C'est un test de serviabilité anonyme. La nuit, les inconnus s'identifient plus rapidement les uns aux autres. Un jeune homme m'a dit : « À 4 heures, si quelqu'un te voit marcher dans la rue en même temps que lui, il doit penser : "Oh, ce mec doit faire partie du club, parce que personne d'autre n'est réveillé à cette heure.” "Cependant, si quelqu'un trouve une clé et ne connaît pas son propriétaire, il présumera que tous ceux qui seront passés par là auront une probabilité équivalente de l'avoir perdue. Comme ils savent qu'ils sont peu nombreux, les noctambules se réfèrent au poids respectif des groupes pour parier que la personne qui a perdu la clé est quelqu'un du jour. Dans la section 10 ci-dessus, je suggérais que les sentiments des noctambules à l'égard des «diurnes » ressemblaient aux attitudes des habitants de l'Ouest vis-à-vis de ceux de l'Est il y a un siècle. Ils perçoivent qu'ils sont différents et s’indignent du mépris exprimé par les gens du jour à leur égard. Les noctambules ont des sentiments de camaraderie envers leurs pairs mais éprouvent de l'indifférence ou de l'hostilité envers les autres ${ }^{66}$. Tandis que les gens des fronts pionniers aident volontiers ceux qu'ils y rencontrent, leur conscience d'une différence avec les diurnes inconnus les rend moins concernés par les soucis de ces derniers et, de fait, ils ne renvoient pas beaucoup de clés perdues.

65. «Dukakis decides to go against Logan curfew », Boston Globe, 12 août 1976, pp. 1 et 20 et« Logan anti-noise plan offered », Boston Globe, 13 août 1976.

66. Sumner W. G., Folkways, New York, New American Library, 1960 [1906], p. 27. 
Je ne peux pas imaginer d'explication concurrente à cette observation qui soit tout aussi plausible, et compatible avec les autres indices. Cette interprétation donne sens à l'ensemble des résultats du tableau 2 et correspond à l'analyse de la précédente section. Quelques tendances saillantes apparaissent. L'une d'entre elles est la connexion entre violence d'un côté, serviabilité et convivialité de l'autre, cette caractéristique des fronts pionniers s'expliquant par la peur et la solidarité entre ceux qui ont le sentiment de partager les mêmes dangers. Une autre tendance est l'association entre esprit communautaire et serviabilité, si bien que l'aide est donnée de façon sélective à ceux avec qui on s'identifie.

Les expériences confirment ce que nous savons de la vie sur les terres nouvellement conquises, mais je n'ai pas exploré pleinement les causes de ces comportements ici. Les constats peuvent être comparés avec les recherches sur l'obligeance présentées par James Bryan et Mary Ann Test (1967), Roy Feldman (1968), Bibb Latané et John Darley (1970), Stanley Milgram (1970), Lauren Wispé et Harold Freshley (1971), John Darley et Daniel Batson (1973) et d'autres encore ${ }^{67}$. Il y a un problème de comparabilité parce que les différents moments du jour n'ont pas été traités de façon systématique dans ces études. Néanmoins certains de leurs résultats apparaissent cohérents. Au moins les découvertes relatives à l'utilisation du temps disponible s'accordentelles les unes avec les autres. Darley et Batson ont fait varier le degré d'urgence avec lequel leurs enquêtés devaient se rendre à un rendez-vous lorsqu'ils tombaient sur une personne toussant, gémissant et ayant apparemment besoin d'aide. Parmi les facteurs pris en compte, qui incluaient les préoccupations des sujets sur le moment (certains d'entre eux se préparaient à discuter de la parabole du Bon Samaritain !), seul le degré d'urgence a été corrélé à l'aide apportée. À peine $10 \%$ de ceux qui étaient en retard à leur rendez-vous se sont arrêtés pour aider, tandis que $63 \%$ de ceux qui avaient suffisamment de temps se sont arrêtés pour apporter de l'aide à l'homme accroupi et souffrant.

67. Bryan J. H. and Test M. A., « Models and helping: naturalistic studies in aiding behavior ", Journal of Personality and Social Psychology, vol. 6, n4, 1967, pp. 400-7 ; Feldman R. E., "Response to compatriot and foreigner who seek assistance ", Journal of Personality and Social Psychology, vol. 10, n³, 1968, pp. 202-214 ; Latané B., Darley J., The Unresponsive Bystander, New York, Appleton-Century, 1970 ; Milgram S., " The experience of living in cities », Science, vol. 167, n³ 3924, 1970, pp. 1461-1468; Wispé L. G. et Freshley H. B., « Race, sex, and sympathetic helping behavior: the broken bag caper ", Journal of Personality and Social Psychology, vol. 17, n 1, 1971, pp. 59-64 ; Darley J., Batson D., "From Jerusalem to Jericho': a study of situational and dispositional variables in helping behavior ", Journal of Personality and Social Psychology, vol. 27, n¹, 1973, pp. 100-108. 


\section{Conclusion}

Quel est l'intérêt de concevoir la nuit comme un front pionnier ? Cette simple proposition théorique donne de la cohérence à une large palette de phénomènes : le type de personnes qui sortent se promener à ces heures ; pourquoi ils diffèrent des gens du jour dans leurs comportements ; les premières initiatives politiques des noctambules; la lente prise de conscience parmi les dirigeants qu'une politique publique peut prendre en compte la ressource temporelle. Même la diversité des projets devient compréhensible - des usines de fusion de métaux aux terrains de mini-golf, en passant par les bureaux municipaux des plaintes, les restaurants, les distributeurs automatiques d'argent. La niche est en train de s'étendre. Petit à petit, toute la société migre vers la nuit. Envisager cela comme la continuation de l'expansion géographique des siècles passés, c'est appréhender le mouvement d'ensemble comme relevant de la migration, de la colonisation et de la frontière, pour reprendre les concepts écologiques usuels.

Bien que je n'aie passé en revue qu'une période de l'histoire des ÉtatsUnis, les mêmes caractéristiques se retrouvent dans toutes les conquêtes territoriales. Elles peuvent s'appliquer au franchissement de l'Oural par les Russes, aux Chinois entrant en Mandchourie durant la dynastie Ch'ing, aux Boers s'installant en Afrique du Sud, aux Australiens s'aventurant dans l'Outback, aux Brésiliens d'aujourd'hui qui colonisent le cœur de l'Amazonie, aussi bien qu'aux Américains qui migrent vers la nuit. Ces modèles sont confirmés par les articles réunis dans le recueil de Wyman et Kroeber sur les frontières.

Nous devons également tenir compte des singularités de cette nouvelle frontière qu'est la nuit. Chaque peuplement au-delà des limites établies a ses propres caractéristiques. Voici quelques différences entre l'Ouest et la nuit : (1) sur les nouvelles terres, les pionniers vivent dans des conditions difficiles avec peu de services à portée de main. La nuit, une large part des activités renvoie à des services. (2) Les équipements sont plus chers sur les marges occidentales ; la nuit, les frais en matière d'appel téléphonique, d'électricité ou de voyage en avion sont moins importants. (3) Tandis que les colonies à l'Ouest étaient isolées de l'Est, jour et nuit sont liés au point que la nuit peut être rapidement affectée par les événements qui se déroulent le jour et vice-versa. La société $24 \mathrm{~h} / 24$ s'adapte continuellement, elle est plus instable. (4) En regardant vers l'Ouest, les pionniers ne voyaient aucune limite aux possibilités de conquête, tandis que nous savons que l'expansion dans la nuit ne peut aller plus loin que l'aube. (5) Les nouvelles terres détenaient la promesse d'une opportunité infinie pour les individus qui s'y aventuraient. Mineurs et pionniers ont enduré des épreuves parce qu'ils vivaient pour le futur. Ils espéraient faire fortune, ou au moins avoir une vie meilleure. La nuit, il y a un grand nombre de tâches non qualifiées, subalternes et sales, mais femme de ménage, 
gardien et aide-soignant sont des emplois sans perspective. Beaucoup de ceux qui occupent ce type d'emploi sont des immigrés ou des membres de minorités et cette marge sociale en expansion est un ghetto temporel. Le ghetto enferme plus que les minorités et les immigrés, puisque les activités de contrôle dans les organisations qui fonctionnent $24 \mathrm{~h} / 24$ reste le fait de l'encadrement supérieur, qui travaille le jour. L'élaboration des politiques, les décisions importantes, l'embauche des employés et les tâches de planification sont réduites pendant les heures creuses. Puisque les équipes du soir et de la nuit ne peuvent réaliser beaucoup des activités qui pourraient conduire à la reconnaissance de leur capacité de direction, et puisque leurs performances ne sont pas directement observables par les chefs, elles ont peu de chances de bénéficier d'avancements. (6) Les ressources naturelles des terres de l'Ouest étaient si étendues que nous sommes devenus des gaspilleurs et nous les avons dilapidées. La nuit, il n'y a rien de nouveau à exploiter à part le temps lui-même, si bien que nous maximisons l'utilisation du capital fixe et devenons plus économes. (7) Les migrations vers l'Ouest supposaient un capital d'investissement relativement important - mises de fond pour une remorque, un équipement minier, du bétail, le chemin de fer. La nuit nécessite peu de capital supplémentaire. Au contraire, le besoin incessant des organisations en termes de personnel reflète une évolution vers des activités à forte densité de main d'œuvre. Ainsi la frontière de la nuit peut-elle faire appel aux pays en développement avec leurs maigres richesses et leur stock impressionnant de chômeurs.

Cette expansion est également inhabituelle parce qu'elle se déroule dans le temps plutôt que dans l'espace. Espèce diurne, nous nous métamorphosons en espèce sans limite temporelle. Nous allons au-delà du cycle environnemental - alternance jour et nuit - dans lequel notre vie biologique et sociale évolue, ce qui est source d'innovations. (8) Dans le passé, une petite équipe de personnes faisait la fermeture d'une entreprise un soir et la relançait le lendemain. Cela facilitait la continuité et la bonne administration. Pour une ouverture en continu, nous avons introduit des rotations de personnel. Plusieurs fois par jour, un autre groupe de personnes prend en charge les mêmes activités et services. (9) Un bouleversement physiologique est imposé aux personnes qui travaillent de nuit et conservent leurs loisirs ordinaires et leur vie sociale lors de leurs jours de repos. Chaque fois qu'ils changent leurs heures de travail, ils subissent un déphasage dans leurs cycles biologiques comme le battement cardiaque, la température et la production hormonale. Le malaise qui en résulte pendant plusieurs jours était connu de ces travailleurs bien avant que les voyages aériens à travers les fuseaux horaires n'aient popularisé l'expression «décalage horaire».

68. Ibsen H., Hedda Gabler (Tr. E. Gosse and W. Archer), in Gassner J. (ed.), A Treasury of the Theatre, New York, Simon and Schuster, 1950 [1890], pp. 42-74, Acte II.

69. Turner F. J., 1893, op. cit., p. 38. 
Le personnage d'Henrik Ibsen, Eilert Lovborg, décrit les deux chapitres du livre qu'il a écrit de la façon suivante : « Le premier traite des [...] forces du futur. Et voici le second prévoyant le développement probable 68 ». Nous pouvons croire que nous comprenons les forces à l'œuvre, les conditions dans lesquelles les humains élargissent leurs niches écologiques, mais quel est le développement probable ? Nous sommes en attente de prévisions, malgré les difficultés de la prospective sociologique. Nous devrions envisager la possibilité d'un futur où les activités en continu seraient même plus fréquentes. Jusqu'où pouvons-nous utiliser les 24 heures d'une journée ? Que se passerat-il lorsqu'il y aura saturation ? La question du temps deviendra de plus en plus cruciale à mesure qu'on le saturera, puisque le temps est une ressource sans substitut direct. Il n'est pas extensible; nous ne pouvons pas agir avec celui-ci comme nous l'avons fait avec la terre en construisant vers le ciel et en creusant dans le sol. Le temps n'est pas stockable; nous ne pouvons conserver les heures non utilisées chaque nuit pour de futurs besoins.

Dans son essai La frontière dans l'histoire américaine, Frederick Jackson Turner analysait l'effet de l'avancée vers les terres de l'Ouest sur notre société et remarquait : "Et aujourd'hui, quatre siècles après la découverte de l'Amérique, une centaine d'années après l'adoption de la Constitution, la frontière a disparu 69 ». Mais elle existe toujours. Alors que la conquête de notre territoire se parachevait, commençait une gigantesque migration d'activités éveillées vers la nuit, qui continue à se déployer dans le monde entier. 